BMJ Open

Diabetes

Research

\& Care

\title{
Effect of bariatric surgery on circulating and urinary mitochondrial DNA copy numbers in obesity with or without diabetes
}

\author{
Mihae Seo, ${ }^{1}$ Hyoungnae Kim (D) , 2,3 Hyunjin Noh, ${ }^{2,3}$ Jin Seok Jeon, ${ }^{2,3}$ \\ Dong Won Byun, ${ }^{2}$ Sang Hyun Kim, ${ }^{4}$ Hye Jeong Kim, ${ }^{2}$ Kyoil Suh, ${ }^{2}$ \\ Hyeong Kyu Park, ${ }^{2}$ Soon Hyo Kwon (1) 2,3
}

To cite: Seo M, Kim H, Noh H, et al. Effect of bariatric surgery on circulating and urinary mitochondrial DNA copy numbers in obesity with or without diabetes. BMJ Open Diab Res Care 2020;8:e001372. doi:10.1136/ bmjdrc-2020-001372

Received 19 March 2020 Revised 22 July 2020 Accepted 23 August 2020

Check for updates

(c) Author(s) (or their employer(s)) 2020. Re-use permitted under CC BY-NC. No commercial re-use. See rights and permissions. Published by BMJ.

${ }^{1}$ Internal Medicine,

Soonchunhyang

University Hospital, Gumi, Gyeongsangbuk-do, The

Republic of Korea

${ }^{2}$ Internal Medicine,

Soonchunhyang University

Seoul Hospital, Seoul, The

Republic of Korea

${ }^{3}$ Hyaonam Kidney Laboratory,

Soonchunhyang University

Seoul Hospital, Seoul, The

Republic of Korea

${ }^{4}$ Department of Surgery,

Soonchunhyang University

Seoul Hospital, Seoul, The

Republic of Korea

Correspondence to

Dr Soon Hyo Kwon;

ksoonhyo@schmc.ac.kr and Dr

Hyeong Kyu Park;

hkpark@schmc.ac.kr

\section{ABSTRACT}

Introduction Recent studies have suggested that extracellular circulating and urinary mitochondrial DNA (mtDNA) are associated with mitochondrial dysfunction in obesity and type 2 diabetes mellitus (T2DM). However, the changes to cell-free serum and urinary mtDNA after bariatric surgery in patients with obesity with T2DM have not been investigated to date.

Research design and methods We prospectively recruited patients with obesity $(n=18)$, and with obesity and T2DM $(n=14)$ who underwent bariatric surgery, along with healthy volunteers $(\mathrm{HV})$ as a control group $(\mathrm{n}=22)$. Serum and urinary mitochondrial nicotinamide adenine dinucleotide dehydrogenase subunit-1 (mtND-1) and cytochrome-c oxidase 3 (mtCOX-3) copy numbers were measured using quantitative PCR (qPCR). The mtDNA copy numbers of patients with obesity (with and without T2DM) were followed up 6 months after surgery.

Results The copy numbers of urinary mtND-1 and mtCOX-3 in patients with obesity, with or without T2DM, were higher than those in the HVs. Moreover, urinary mtCOX-3 copy number increased in patients with obesity with T2DM compared with patients with obesity without T2DM ( $p=0.018)$. Meanwhile, serum mtCOX-3 copy numbers in HV were higher in both obesity patient groups $(p=0.040)$. Bariatric surgery reduced urinary mtND-1 and mtCOX-3 copy numbers, as well as serum mtCOX-3 copy numbers only in patients with obesity with T2DM. Conclusion These results suggest that T2DM induces greater kidney mitochondrial dysfunction in patients with obesity, which can be effectively restored with bariatric surgery.

\section{INTRODUCTION}

Obesity is associated with oxidative stress, which can result in mitochondrial damage and a defect in mitochondrial biogenesis. ${ }^{1-6}$ Triggers, such as hyperglycemia, can induce increased intracellular reactive oxygen species (ROS).$^{1-7}$ Surprisingly, bariatric surgery-induced weight loss has been shown to improve mitochondrial biogenesis, whereas diet-induced weight reduction did

\section{Significance of this study}

What is already known about this subject?

- Extracellular circulating and urinary mitochondrial DNA (mtDNA) are reportedly associated with mitochondrial dysfunction in obesity and type 2 diabetes mellitus (T2DM).

- Bariatric surgery could improve mitochondrial dysfunction in obesity.

- Bariatric surgery-induced weight loss improves mitochondrial biogenesis, whereas diet-induced weight loss did not improve mitochondrial dysfunction.

What are the new findings?

- Obesity does not increase circulating mtDNA copy numbers.

- Diabetic effect on kidney mitochondrial respiratory chain (MRC) is likely prominent in MRC-IV (mitochondrial cytochrome-c oxidase 3) in patients with obesity.

- Bariatric surgery restores kidney mitochondrial dysfunction in patients with obesity with T2DM.

How might these results change the focus of research or clinical practice?

- Improving T2DM and nephropathy after bariatric surgery can also affect serum and urinary mtDNA copy numbers, further highlighting that T2DM is strongly associated with mitochondrial dysfunction in patients with obesity.

- Promotion of bariatric surgery should be considered in individuals with obesity with kidney disease or T2DM.

not improve mitochondrial dysfunction. Moreover, bariatric surgery may be associated with improved renal tubular injury, which is determined via monitoring the level of kidney injury molecule-1 in patients with obesity. ${ }^{8}$ This finding may account for the additional benefits experienced by patients after bariatric surgery, independent of weight reduction. For example, bariatric surgery 
in patients with obesity involves weight-independent glucose-lowering effects, and is thus considered to be a highly effective method for preventing, or resolving, type 2 diabetes mellitus (T2DM), regardless of the patient's T2DM status prior to surgery. ${ }^{10}$ Numerous studies have also reported that bariatric surgery is superior for improving glycemic and metabolic variables compared with non-surgical treatments in patients with obesity with T2DM. ${ }^{1011}$ A Danish study further showed that individuals with obesity with T2DM had a 47\% lower risk of microvascular complications after bariatric surgery compared with that of patients that did not receive the surgery. ${ }^{11}$ In addition, bariatric surgery significantly reduced genomic damage in an obese rat model. ${ }^{12}$ However, the underlying mechanism remains unclear, and despite the known link between mitochondrial damage and obesity, the influence of bariatric surgery on changes to cell-free mitochondrial DNA (mtDNA) in patients with obesity with T2DM has not been established.

Mitochondria contain their own DNA genome ${ }^{1}$; thus, the mtDNA copy number can reflect the degree of mitochondrial damage. The cell-free mtDNA copy number has been explored as an easily accessible and non-invasive biomarker for the detection of mitochondrial dysfunction, ${ }^{1314}$ which is generally measured as the mitochondrialto-nuclear genome ratio $(\mathrm{Mt} / \mathrm{N}) .{ }^{1}$ The intracellular and extracellular mtDNA levels have been suggested as associated with various clinical outcomes. ${ }^{2315}$ Cell-free mtDNA is reportedly derived from injured tissue or cells in the body. ${ }^{16}$ Specifically, urinary cell-free mtDNA content has been most widely evaluated in various clinical settings as a non-invasive biomarker, of which increased levels can effectively predict poor outcomes in patients with T2DM, acute kidney injury and diabetic nephropathy. ${ }^{15}$

We previously observed decreased urinary mtDNA levels after bariatric surgery in patients with obesity and without diabetes. ${ }^{17}$ In addition, the intracellular mtDNA copy number recovered in patients with obesity after weight reduction surgery, reaching the level of nonobese control subjects. ${ }^{18}$ However, since T2DM induces higher degrees of mitochondrial dysfunction in patients with obesity, ${ }^{418}$ the extracellular mtDNA copy number in patients with obesity with T2DM may differ from that of patients without diabetes.

Thus, the aim of the present study was to explore the change in cell-free serum and urine mtDNA copy numbers in patients with morbid obesity with, and without, T2DM before, and after, bariatric surgery as a surrogate for mitochondrial dysfunction, and the levels were compared with those of healthy volunteers (HV) as a control group.

\section{RESEARCH DESIGN AND METHODS}

\section{Subjects}

We prospectively recruited HV $(n=22)$, patients with obesity $(\mathrm{n}=18)$ and patients with obesity and T2DM $(n=14)$ who were scheduled to undergo bariatric surgery at Soonchunhyang University Hospital (Seoul, Republic of Korea). The inclusion criteria for patients with obesity were: bariatric surgery candidates, body mass index (BMI) $>35$ or $>30 \mathrm{~kg} / \mathrm{m}^{2}$ for those with comorbidities related to obesity such as hypertension (HTN), obstructive sleep apnea and obesity-related arthropathy. ${ }^{17}$ The specific surgical procedure performed was determined on an individual basis after interdisciplinary discussion with consideration of relevant patient information. Exclusion criteria were as follows: those who had experienced major cardiovascular or cerebrovascular events in the 6 months preceding the study, those who had undergone kidney transplantation and those with a serum creatinine (Cr) level $>2.5 \mathrm{mg} / \mathrm{dL}$. The inclusion criteria for $\mathrm{HVs}$ were: 18 years or older, not taking any medication and no history of HTN, T2DM, congestive heart failure, cardiovascular disease or cerebrovascular disease.

\section{Anthropometric and biochemical assessments}

We collected the demographic information and laboratory measurements of the participants at the start of the study. The estimated glomerular filtration rate (eGFR) was calculated using the CKD Epidemiology Collaboration (CKD-EPI) equation ${ }^{19}$ and serum cystatin C. Urine samples from all participants were collected over a 24 hours period. After collection, the samples were centrifuged, and the supernatants were collected and frozen at $-80^{\circ} \mathrm{C}$ until analysis. The follow-up data for patients with obesity, with and without T2DM, were collected 6 months after bariatric surgery. Serum and urine mtDNA copy numbers were measured in HVs and presurgery patients, and re-analysed 6 months after the surgery.

\section{Measurement of mitochondrial DNA copy number}

COX 3 encodes the terminal enzyme of the mitochondrial respiratory chain (MRC)-IV, which catalyses the transfer of electrons from reduced cytochrome-c to oxygen; whereas ND1 encodes one of the subunits of MRC-I, an enzyme responsible for the first step in the electron transport chain during with electrons are transferred from NADH to ubiquinone. ${ }^{20}$ Furthermore, these genes are located at sites opposite each other on the circular mtDNA and may, therefore, represent mtDNA functionally and anatomically in particular. ${ }^{20}$

The urinary and serum mtDNA content of all participants was measured according to the copy numbers of mitochondrial nicotinamide adenine dinucleotide dehydrogenase subunit-1 (mtND-1) and cytochrome-c oxidase3 (mtCOX-3) using quantitative PCR (qPCR). DNA was isolated and purified from urine samples $(1.75 \mathrm{~mL})$ and serum $(200 \mathrm{~mL})$ using DNA isolation kits from Norgen Biotek (Thorold, Ontario, Canada; Catalog Number 18100) according to the manufacturer's instructions. The DNA concentration was measured using a Nano Drop spectrophotometer (Thermo Fisher Scientific, Waltham, Massachusetts, USA). qPCR was performed with $20 \mathrm{ng}$ of template DNA/sample and the following primers: ND1 forward, 5'-AGTCACCCTAGC 
Table 1 Demographic characteristics and laboratory measurements of study participants

\begin{tabular}{|c|c|c|c|c|c|}
\hline & $\begin{array}{l}\text { Healthy volunteers } \\
\mathrm{n}=22\end{array}$ & $\begin{array}{l}\text { Patients with obesity } \\
n=18\end{array}$ & P valuet & $\begin{array}{l}\text { Patients with obesity with } \\
\text { T2DM } n=14\end{array}$ & $P$ value $¥$ \\
\hline Age, (year) & $35.23 \pm 9.60$ & $30.89 \pm 8.44$ & 0.142 & $42.00 \pm 12.39$ & 0.005 \\
\hline Weight, kg & $59.72 \pm 8.52$ & $118.1 \pm 26.89$ & $<0.001$ & $114.36 \pm 32.44^{*}$ & 0.724 \\
\hline BMI, $\mathrm{kg} / \mathrm{m}^{2}$ & $21.92 \pm 1.62$ & $40.94 \pm 5.97$ & $<0.001$ & $40.34 \pm 9.02^{*}$ & 0.824 \\
\hline WBC, $\mu \mathrm{L}$ & $5550 \pm 1228.92$ & $8055.56 \pm 2130.24$ & $<0.001$ & $9385.71 \pm 2342.21^{*}$ & 0.104 \\
\hline $\begin{array}{l}\text { eGFR CKD-EPI by Cr, mL/ } \\
\min / 1.73 \mathrm{~m}^{2}\end{array}$ & $108.16 \pm 11.73$ & $116.27 \pm 10.65$ & 0.029 & $107.3 \pm 37.24$ & 0.396 \\
\hline $\begin{array}{l}\text { eGFR CKD-EPI by cysC, } \\
\mathrm{mL} / \mathrm{min} / 1.73 \mathrm{~m}^{2}\end{array}$ & $116.82 \pm 10.89$ & $105.86 \pm 10.47$ & 0.003 & $93.65 \pm 37.46^{\star}$ & 0.256 \\
\hline Triglyceride mg/dL & $93.38 \pm 52.52$ & $193.28 \pm 115.84$ & 0.003 & $205.57 \pm 70.61^{*}$ & 0.729 \\
\hline LDL cholesterol mg/dL & $106.32 \pm 19.6$ & $148.67 \pm 41.13$ & 0.001 & $118.33 \pm 49.76$ & 0.069 \\
\hline HDL cholesterol mg/dL & $66.73 \pm 14.23$ & $46.83 \pm 9.61$ & $<0.001$ & $45.91 \pm 15.98^{\star}$ & 0.840 \\
\hline Glucose, mg/dL & $97.05 \pm 7.12$ & $107.61 \pm 16.26$ & 0.018 & $148.58 \pm 51.09^{*}$ & 0.011 \\
\hline Cystatin C, mg/L & $0.78 \pm 0.09$ & $0.86 \pm 0.09$ & 0.005 & $3 \pm 7.21$ & 0.288 \\
\hline C-peptide, ng/mL & & $4.54 \pm 1.97$ & $\mathrm{~N} / \mathrm{A}$ & $3.72 \pm 1.63$ & 0.215 \\
\hline $\mathrm{BUN}, \mathrm{mg} / \mathrm{dL}$ & $11.2(8.88-12.9)$ & 10.85 (9.58-13.35) & 0.744 & $14.35(11.08-20.98)^{*}$ & 0.055 \\
\hline Serum Cr, mg/dL & $0.72(0.67-0.99)$ & $0.67(0.61-0.87)$ & 0.165 & 0.745 (0.56-0.94) & 0.732 \\
\hline
\end{tabular}

Data are presented as means \pm SD or median $(\mathrm{IQR})$. Differences among healthy volunteers, patients with obesity and patients with obesity with T2DM were assessed using Student's t-test and Mann-Whitney $U$ test. The sex variables were evaluated using $\chi^{2}$ test. ${ }^{*} \mathrm{P}<0.05$ between healthy volunteer and patients with obesity with T2DM.

†P (healthy volunteers vs patients with obesity).

ҒP (patient with obesity vs patients with obesity with T2DM).

ALT, alanine aminotransferase; AST, aspartate aminotransferase; BMI, body mass index; BP, blood pressure; BSA, body surface area; BUN, blood urea nitrogen; CKD-EPI, CKD Epidemiology Collaboration; Cr, creatinine; eGFR, estimated glomerular filtration rate; HbA1c, hemoglobin A1c; HDL, high-density lipoprotein; LDL, low-density lipoprotein; N/A, not available; T2DM, type 2 diabetes mellitus; WBC, white blood cell.

CATCATTCTACT-3', and reverse, 5'-GGAGTAATCAGA GGTGTTCTTGTGT-3'; COX3 forward, 5'-AGGCATCACCCCGCTAAATC-3', and reverse, 5'-GGTGAGCT CAGGTGATTGATACTC-3' (Thermo Fisher Scientific, Carlsbad, California, USA). The PCR conditions were as follows: $95^{\circ} \mathrm{C}$ for $10 \mathrm{~min}, 40$ cycles of $95^{\circ} \mathrm{C}$ for $15 \mathrm{~s}$ and $60^{\circ} \mathrm{C}$ for $60 \mathrm{~s}$. To calculate the absolute DNA copy numbers in each sample, serial dilutions were created for the COX3 (Blue Heron BioTechnology, Bothell, Massachusetts, USA; NC0129201) and ND1 (OriGene, Rockville, Maryland, USA; SC101172) plasmid constructs and run together with the samples. To identify mitochondriaspecific cellular damage, mtDNA copy numbers were corrected to those of the nuclear DNA control gene, RNaseP (Thermo Fisher Scientific; Catalog Number
4403326) using human genomic DNA for the standard curve. The copy numbers were determined using Copy Caller software (Thermo Fisher Scientific) and expressed as the $\mathrm{Mt} / \mathrm{N}$ ratio. ${ }^{21}$

\section{Statistical analysis}

All statistical analyses were performed using the commercially available SPSS V.14.0 software package (SPSS, Chicago, Illinois, USA). For continuous data, normally distributed data are expressed as means \pm SD and were compared between groups using Student's t-test. Nonnormally distributed data are expressed as the median (IQR) and were compared using the Mann-Whitney U test. Categorical data are expressed as proportions and were compared using the $\chi^{2}$ test. Correlations were 
Table 2 Baseline characteristics of study population before and after bariatric surgery

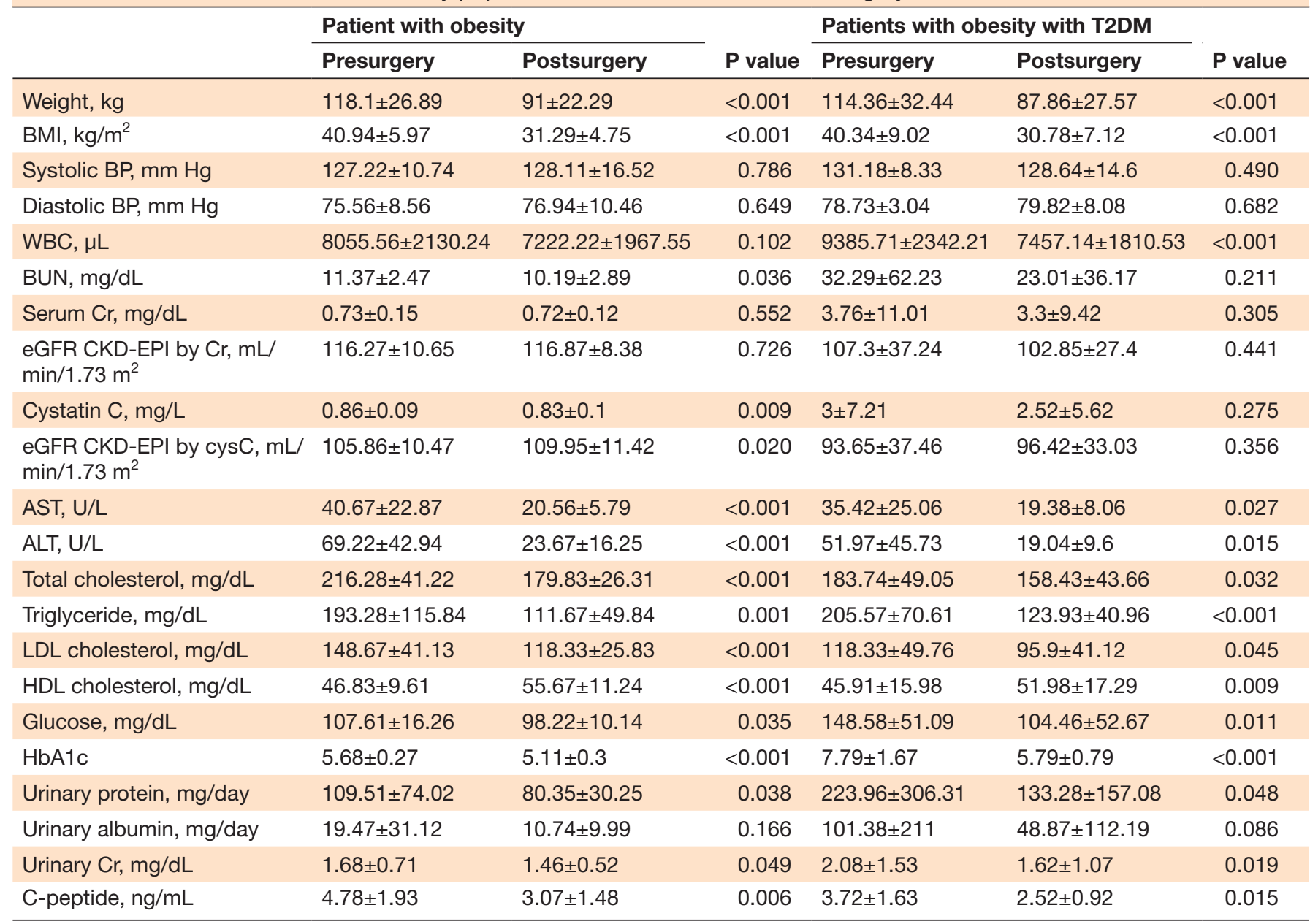

Data are presented as means \pm SD or median (IQR). Differences between patients with obesity and patients with obesity with T2DM were assessed using Student's t-test and Mann-Whitney $U$ test. The sex variables were evaluated using $\chi^{2}$ test.

ALT, alanine aminotransferase; AST, aspartate aminotransferase; BMI, body mass index; BP, blood pressure; BSA, body surface area; BUN, blood urea nitrogen; CKD-EPI, CKD Epidemiology Collaboration; Cr, creatinine; eGFR, estimated glomerular filtration rate; HbA1c, hemoglobin A1c; HDL, high-density lipoprotein; LDL, low-density lipoprotein; T2DM, type 2 diabtes mellitus; WBC, white blood cell.

determined by the Pearson's correlation coefficient. Longitudinal data were compared using the paired t-test for normally distributed data and using the Wilcoxon signed-rank test for non-normally distributed data. A p value of $<0.05$ was considered to be statistically significant.

\section{RESULTS}

\section{Participant characteristics}

The baseline characteristics and laboratory measurements of the participants are presented in table 1. Patients with obesity with, and without T2DM, had metabolically unfavorable laboratory findings compared with the HV. Patients with obesity and T2DM were older on average than the other two groups. The sex ratio and eGFR did not significantly differ between groups. Compared with patients with obesity, patients with obesity and T2DM had higher total cholesterol, glucose, hemoglobin A1c (HbAlc), urinary protein and urinary albumin levels. However, BMI did not differ between the two obese groups.

\section{Effect of bariatric surgery on clinical parameters}

Table 2 presents the comparisons of clinical parameters before and after bariatric surgery in patients with obesity, with and without T2DM. Patients with obesity with T2DM had higher glucose, HbA1c, urinary protein and urinary albumin levels at baseline compared with those without T2DM. The mean BMI was reduced from 40.64 to $31.04 \mathrm{~kg} / \mathrm{m}^{2}$, and the mean body weight reduced from 116.23 to $89.43 \mathrm{~kg}$ in all patients with obesity 6 months after bariatric surgery. In addition, patients with obesity with T2DM showed significantly reduced fasting glucose levels, HbA1c and low-density lipoproteincholesterol levels 6 months after surgery compared with the baseline values. By contrast, there was no change in the fasting glucose or HbAlc levels of the patients with obesity without diabetes after surgery. There was also a significant reduction in the 24 hours urinary protein and urinary albumin levels in patients with obesity with T2DM. However, the eGFR was not affected by surgery in either of the patient groups. 
(A)
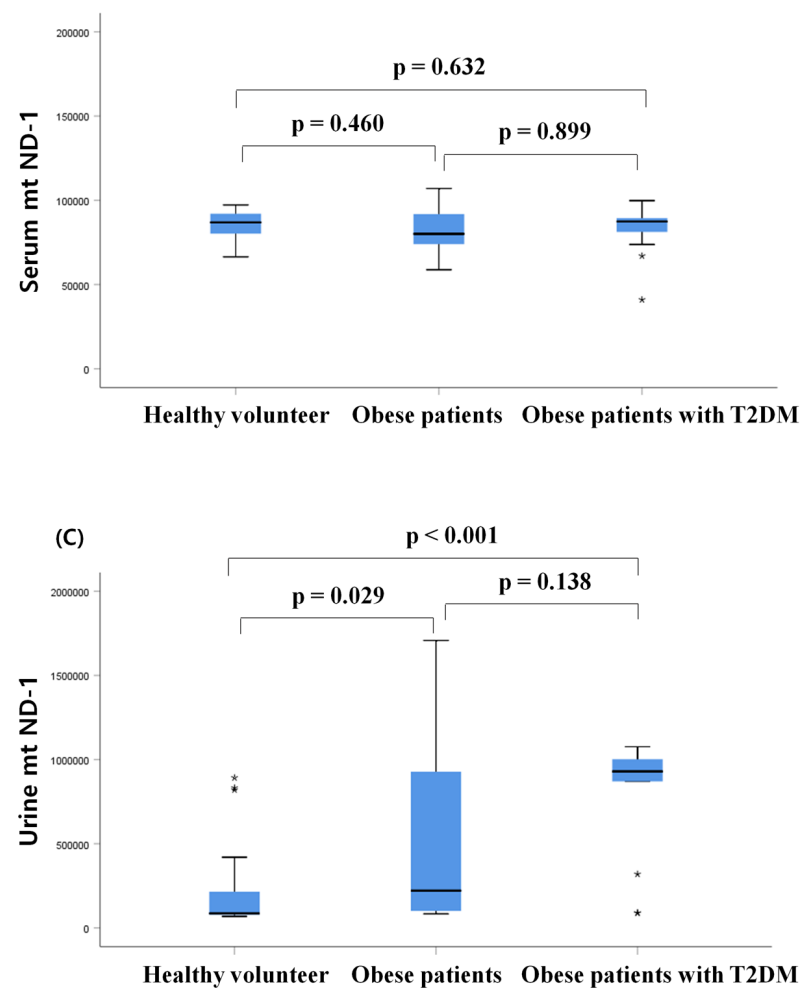

(B)
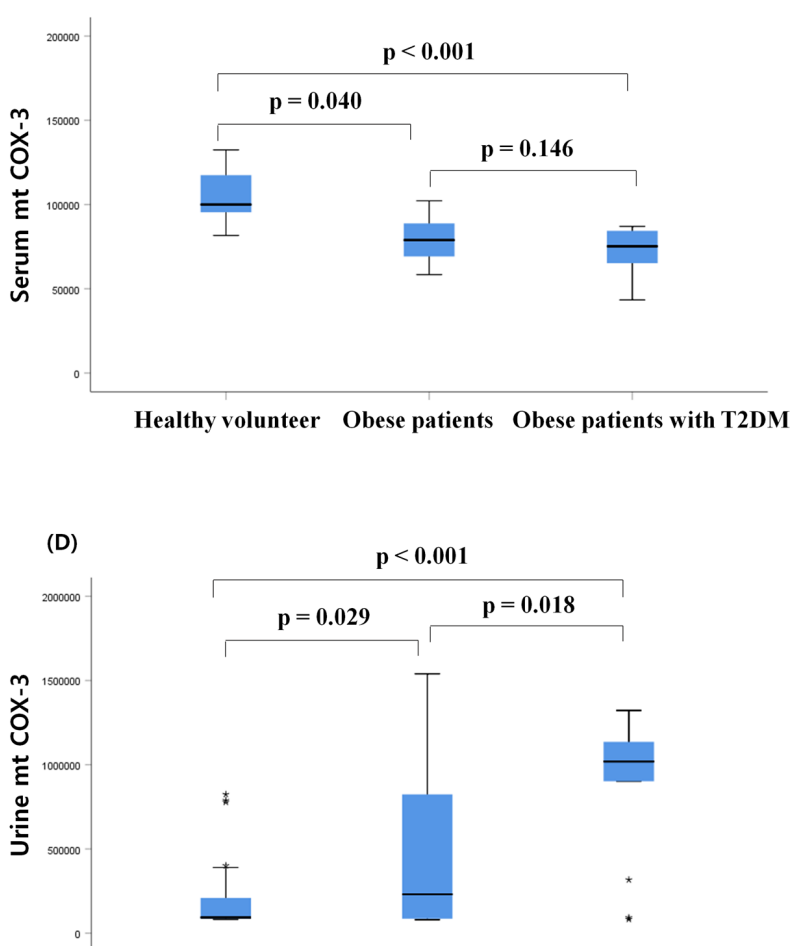

Healthy volunteer Obese patients Obese patients with T2DM

Figure 1 Comparison of serum and urine mitochondrial DNA (mtDNA) copy number at baseline. (A) Serum mitochondrial nicotinamide adenine dinucleotide dehydrogenase subunit-1 (mtND-1), (B) serum cytochrome-c oxidase 3 (COX-3), (C) urine mtND-1, (D) urine mitochondrial COX-3 (mtCOX-3) in healthy volunteers, patients with obesity and patients with obesity with type 2 diabetes mellitus (T2DM) group at baseline. Box represents first and third quartile and median. Statistical differences between groups were analyzed by analysis of variance followed by Fisher's LSD test. LSD, least significant difference.

Baseline differences in serum and urine mtDNA levels among HV, and among patients with obesity and patients with obesity with T2DM

The serum mtND-1 copy number at baseline did not differ between the three groups (figure 1). However, the serum mtCOX-3 copy number of patients with obesity was reduced compared with that of $\mathrm{HV}$, regardless of T2DM status.

The urinary mtND-1 and mtCOX-3 copy numbers were also significantly higher in patients with obesity, with or without T2DM, compared with those of HV. The urinary mtCOX-3 copy number in patients with obesity with T2DM increased compared with patients with obesity without diabetes.

\section{Changes in mtDNA copy numbers after bariatric surgery}

In all individuals with obesity, with or without T2DM, bariatric surgery decreased serum COX-3 copy number $(\mathrm{p}=0.039)$, as well as urinary ND-1 and COX-3 copy numbers $(p=0.006, p=0.030$, respectively). However, the change in mtDNA copy number differed depending on the DM status (figure 2). Specifically, bariatric surgery did not affect the serum mtND-1 in patients with obesity with and without diabetes, whereas serum COX-3 copy number in patients with obesity with diabetes decreased after surgery (figure 2A,B). Moreover, following bariatric surgery, the urinary mtDNA copy numbers for both
mtND-1 and COX-3 decreased in patients with obesity with diabetes (figure 2C,D).

\section{Correlation between bariatric surgery-induced weight loss and serum and urinary mtDNA copy numbers}

Correlation analysis showed no association between the change in serum and urinary mtDNA copy numbers with changes in other metabolic variables, including bariatric surgery-induced weight loss, in patients with obesity (tables 3 and 4). However, in patients with obesity with T2DM, the weight loss-induced changes in serum mtND-1 and mtCOX-3 copy numbers were positively correlated with the change in diastolic blood pressure and HbAlc levels (table 4). In addition, the changes in urinary mtND-1 and mtCOX-3 copy numbers were associated with changes in eGFR of patients with obesity with T2DM (table 4). However, there were no correlations observed between the changes in the other metabolic parameters, including weight loss, with serum and urine mtDNA copy numbers in patients with obesity with T2DM (table 4).

\section{DISCUSSION}

Herein, we have demonstrated that obesity affects the serum and urinary mtDNA copy numbers regardless of T2DM status. However, diabetes affected the expression of urinary mtCOX-3 in obesity, suggesting that sustained 
(A)

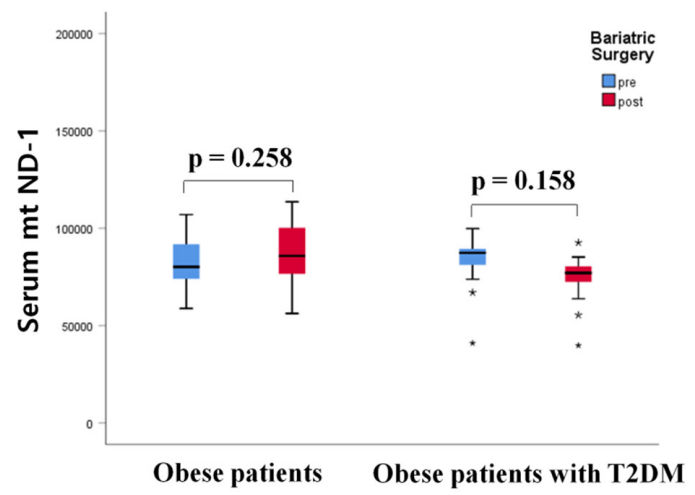

(C)

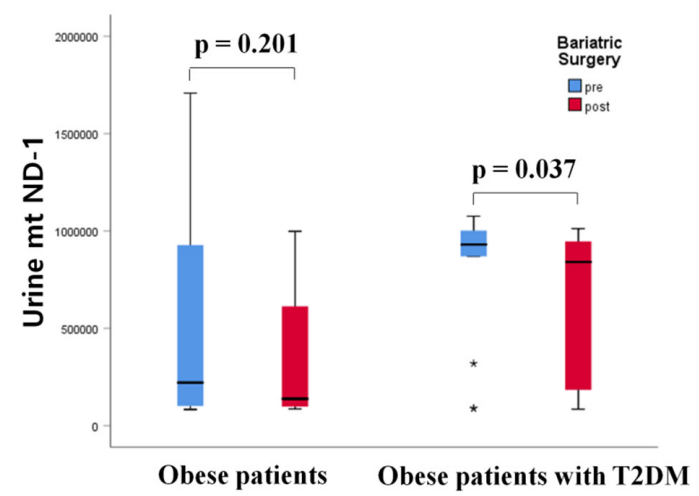

(B)

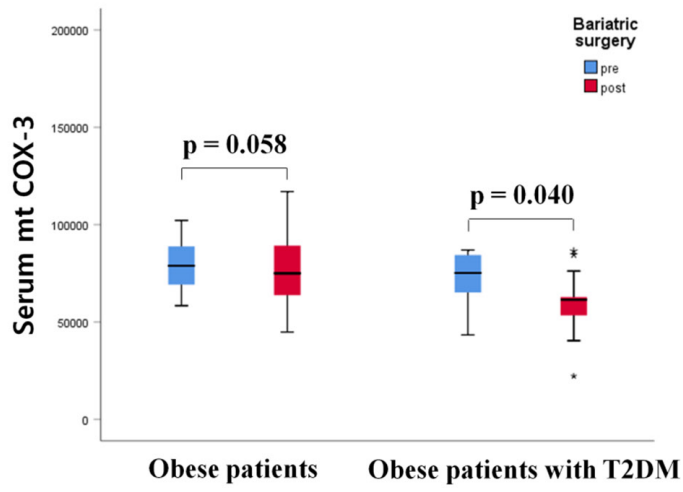

(1)

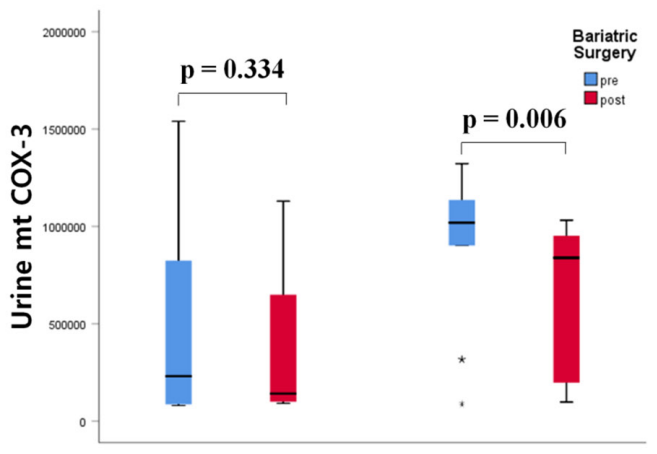

Obese patients Obese patients with T2DM

Figure 2 Changes in serum and urinary mitochondrial DNA (mtDNA), (A) Serum mitochondrial nicotinamide adenine dinucleotide dehydrogenase subunit-1 (mtND-1), (B) serum cytochrome-c oxidase 3 (COX-3), (C) urine mtND-1, (D) urine mitochondrial COX-3 (mtCOX-3) in patients with obesity and patient with obesity with type 2 diabetes mellius (T2DM) after bariatric surgery.

hyperglycemia may lead to increased MRC-IV (mtCOX-3) damage. In addition, weight reduction associated with bariatric surgery reduced circulating mtCOX-3 and urinary mtDNA copy numbers in patients with obesity with diabetes, however, did not have the same effect in patients with obesity without diabetes.

Although the pattern of urinary mtCOX-3 was similar to that of mtND-1, the behavior differed according to T2DM presence. Specifically, only urinary mtCOX-3 copy number increased in a stepwise manner, from HV to patients with obesity without T2DM, to patients with obesity with DM. This finding suggests that obesity induced mitochondrial dysfunction, which can be aggravated by sustained hyperglycemia, such as the case in T2DM. This is consistent with a previous study showing that impaired mitochondrial function in the adipose tissue was associated with acquired obesity without T2DM. ${ }^{5}$ In addition, mitochondrial density in the subcutaneous cells of diabetic patients with obesity was reported to be lower than that of non-diabetic patients with obesity. ${ }^{4}$ However, the damage to the MRC induced by obesity may differ according to the specific organ, such as adipocytes or liver. ${ }^{22}$ Since the urinary mtND-1 copy number did not increase in diabetic patients with obesity, our results suggest that DM may have a greater effect on mtCOX-3 levels. ${ }^{22}$

Interestingly, the circulating mtCOX-3 copy number was lower in diabetic and non-diabetic patients with obesity compared with the HV group at baseline, while a greater decrease was observed in this factor following bariatric surgery in diabetic patients compared with non-diabetic patients. This finding is consistent with a previous study that explored the relationship between plasma mtDNA and urinary mtDNA in patients with diabetes and animal models. ${ }^{15}$ The plasma mtDNA level of patients with T2DM was lower than that of healthy controls, and the urinary mtDNA correlated with proteinuria in patients with T2DM. ${ }^{15}$ The authors suggested that the kidney was responsible for reducing plasma mtDNA levels via hyperfiltration. However, this interpretation disagrees with our present and previous findings, which show that hyperfiltration would resolve after bariatric surgery. ${ }^{23} 24$ According to their hypothesis, the mtDNA copy number would be expected to decrease after bariatric surgery. ${ }^{2324}$

Although bariatric surgery decreased serum and urinary mtDNA copy numbers in diabetic patients, no such reduction was observed in the non-diabetic patients with obesity. Obesity induces glomerular hyperfiltration, 
Table 3 Univariate analysis for correlations between weight loss-induced changes in serum and urine mtND-1 and mtCOX-3 copy number and the changes in other variables in patients with obesity

\begin{tabular}{|c|c|c|c|c|c|c|c|c|}
\hline \multirow[b]{2}{*}{ Variables $(\Delta)$} & \multicolumn{2}{|c|}{$\Delta$ serum mtND-1 } & \multicolumn{2}{|c|}{$\Delta$ serum mtCOX-3 } & \multicolumn{2}{|c|}{$\Delta$ urine mtND-1 } & \multicolumn{2}{|c|}{$\Delta$ urine mtCOX-3 } \\
\hline & $r$ & $P$ value & $\mathbf{r}$ & $P$ value & $\mathbf{r}$ & $P$ value & $r$ & $P$ value \\
\hline Weight, kg & -0.046 & 0.769 & 0.024 & 0.877 & -0.176 & 0.254 & -0.109 & 0.480 \\
\hline BMI, $\mathrm{kg} / \mathrm{m}^{2}$ & 0.119 & 0.446 & 0.070 & 0.655 & 0.210 & 0.172 & 0.150 & 0.332 \\
\hline Systolic BP, mm Hg & 0.171 & 0.318 & 0.064 & 0.712 & 0.214 & 0.203 & 0.143 & 0.399 \\
\hline Diastolic BP, mm Hg & $0.362^{*}$ & 0.030 & 0.272 & 0.108 & 0.109 & 0.522 & 0.108 & 0.524 \\
\hline $\begin{array}{l}\text { eGFR CKD-EPI by cysC, } \mathrm{mL} / \mathrm{min} / 1.73 \\
\mathrm{~m}^{2}\end{array}$ & $-0.360^{*}$ & 0.047 & $-0.357^{*}$ & 0.049 & 0.225 & 0.216 & 0.234 & 0.198 \\
\hline AST & 0.128 & 0.445 & 0.061 & 0.716 & 0.027 & 0.871 & 0.042 & 0.801 \\
\hline ALT & 0.121 & 0.468 & -0.032 & 0.848 & 0.023 & 0.889 & 0.073 & 0.659 \\
\hline Triglyceride, mg/dL & 0.080 & 0.642 & 0.136 & 0.428 & -0.270 & 0.106 & -0.290 & 0.082 \\
\hline LDL cholesterol, mg/dL & -0.006 & 0.969 & -0.080 & 0.628 & -0.067 & 0.680 & -0.071 & 0.661 \\
\hline HDL cholesterol, mg/dL & -0.016 & 0.927 & -0.042 & 0.816 & -0.004 & 0.983 & -0.039 & 0.826 \\
\hline Glucose, mg/dL & 0.091 & 0.582 & 0.067 & 0.686 & 0.052 & 0.750 & -0.015 & 0.929 \\
\hline $\mathrm{HbA1c}$ & -0.003 & 0.985 & -0.039 & 0.806 & 0.048 & 0.758 & 0.035 & 0.826 \\
\hline Urinary protein, mg/day & -0.250 & 0.175 & -0.303 & 0.097 & -0.040 & 0.829 & -0.076 & 0.679 \\
\hline Urinary albumin, mg/day & -0.222 & 0.230 & -0.233 & 0.208 & -0.078 & 0.679 & -0.114 & 0.540 \\
\hline
\end{tabular}

Values are Pearson's correlation coefficients and associated $\mathrm{p}$ values. Values in bold indicate statistically significant correlation at a value of $p<0.05$.

${ }^{*} \mathrm{P}<0.05,{ }^{* *} \mathrm{p}<0.005$.

ALT, alanine transaminase; AST, aspartate transaminase; BMI, body mass index; BP, blood pressure; CKD-EPI, CKD Epidemiology Collaboration; eGFR, estimated glomerular filtration rate; HbA1c, hemoglobin A1c; HDL, high-density lipoprotein; LDL, lowdensity lipoprotein; mtCOX-3, mitochondrial cytochrome-c oxidase 3; mtND-1, mitochondrial nicotinamide adenine dinucleotide dehydrogenase subunit-1.

whereas T2DM itself affects the cellular distribution, and this change can also affect the cell-free mtDNA content. $^{121}$

Neither the change in serum mtND-1, nor mtCOX-3 copy number, correlated with bariatric surgery-induced weight loss. In addition, the change in urinary mtND-1 and COX-3 copy numbers did not correlate with bariatric surgery-induced weight loss or changes in metabolic parameters. However, the change in serum mtDNA copy number was significantly correlated with the change in HbA1c levels, and the change in urinary mtDNA copy number was correlated with the change in eGFR in patients with obesity with T2DM. These results suggest that improving T2DM and nephropathy after bariatric surgery can also affect serum and urinary mtDNA copy numbers, further highlighting that T2DM is strongly associated with mitochondrial dysfunction in patients with obesity

There are several possible explanations for the greater change observed in the mtDNA copy number in patients with obesity with T2DM, compared with those without T2DM after surgery. First, an altered mtDNA copy number reflects the degree of oxidant-induced cell damage. Obesity is characterized by chronic low-grade inflammation with increased oxidative stress; meanwhile hyperglycemia also induces the overproduction of ROS leading to DNA single-strand breaks. ${ }^{25}$ Moreover, the coexistence of obesity with T2DM significantly contributes to the production of excess ROS, thereby aggravating oxidative stress. ${ }^{25}$ Second, the mitochondrial dysfunction-triggered activation of inflammatory signaling pathways may serve as a potential mechanism underlying obesity-induced insulin resistance. ${ }^{26}$ Under certain pathophysiological conditions, increased mitochondrial stress leads to ROS overproduction, as well as to mtDNA oxidation, and packaging disturbance, triggering the release of mtDNA to the cytoplasm. ${ }^{26}$ However, the specific pathways and mechanisms contributing to obesity-induced mtDNA release, inflammation and hyperglycemia remain unexplored. Our study is the first to compare mtDNA changes in patients with obesity, with and without T2DM, after bariatric surgery, demonstrating a great impact of diabetic status.

Certain limitations were noted in our study. First, only a small number of patients and volunteers were enrolled, and the patients were followed up for a short period of time. Second, patients with obesity with T2DM were older on average than the subjects in the other groups. Therefore, we could not exclude the possibility of an agerelated effect on baseline mtDNA copy numbers.

In conclusion, our study suggests that mitochondrial dysfunction in patients with obesity is likely prominent in a persistent hyperglycemic condition. T2DM might induce greater mitochondrial dysfunction in patients with obesity, which could be effectively restored with bariatric surgery. We further demonstrated that bariatric 
Table 4 Univariate analysis for correlations between weight loss-induced changes in serum and urine mtND-1 and mtCOX-3 copy number and the changes in other variables in patients with obesity with T2DM

\begin{tabular}{|c|c|c|c|c|c|c|c|c|}
\hline \multirow[b]{2}{*}{ Variables $(\Delta)$} & \multicolumn{2}{|c|}{$\Delta$ serum mtND-1 } & \multicolumn{2}{|c|}{$\Delta$ serum mtCOX-3 } & \multicolumn{2}{|c|}{$\Delta$ urine mtND-1 } & \multicolumn{2}{|c|}{$\Delta$ urine mtCOX-3 } \\
\hline & $r$ & $P$ value & $r$ & $P$ value & $r$ & $P$ value & $r$ & $P$ value \\
\hline Weight, kg & 0.231 & 0.448 & -0.048 & 0.875 & -0.237 & 0.415 & -0.105 & 0.721 \\
\hline $\mathrm{BMI}, \mathrm{kg} / \mathrm{m}^{2}$ & -0.108 & 0.726 & 0.285 & 0.345 & 0.350 & 0.220 & 0.167 & 0.569 \\
\hline Systolic BP, mm Hg & 0.545 & 0.104 & 0.561 & 0.092 & 0.202 & 0.551 & 0.142 & 0.677 \\
\hline Diastolic BP, mm Hg & $0.761^{*}$ & 0.011 & $0.744^{*}$ & 0.014 & 0.160 & 0.638 & 0.294 & 0.380 \\
\hline $\begin{array}{l}\text { eGFR CKD-EPI by cysC, mL/min/1.73 } \\
\mathrm{m}^{2}\end{array}$ & -0.401 & 0.174 & -0.331 & 0.270 & $0.539^{*}$ & 0.047 & $0.548^{*}$ & 0.043 \\
\hline AST & -0.110 & 0.721 & -0.044 & 0.886 & -0.527 & 0.053 & -0.504 & 0.066 \\
\hline ALT & -0.070 & 0.821 & -0.215 & 0.481 & -0.487 & 0.078 & -0.344 & 0.229 \\
\hline Triglyceride, $\mathrm{mg} / \mathrm{dL}$ & 0.246 & 0.419 & 0.399 & 0.177 & 0.249 & 0.391 & 0.189 & 0.517 \\
\hline LDL cholesterol, mg/dL & -0.212 & 0.488 & -0.228 & 0.454 & -0.059 & 0.841 & -0.179 & 0.540 \\
\hline HDL cholesterol, mg/dL & -0.008 & 0.978 & -0.223 & 0.464 & -0.014 & 0.961 & 0.024 & 0.936 \\
\hline Glucose, mg/dL & 0.454 & 0.119 & 0.383 & 0.196 & 0.074 & 0.801 & 0.067 & 0.821 \\
\hline $\mathrm{HbA} 1 \mathrm{c}$ & $0.708^{* *}$ & 0.007 & $0.555^{*}$ & 0.049 & 0.089 & 0.762 & 0.138 & 0.639 \\
\hline Urinary protein, mg/day & -0.122 & 0.691 & -0.281 & 0.352 & -0.025 & 0.931 & -0.003 & 0.993 \\
\hline Urinary albumin, mg/day & -0.170 & 0.579 & -0.250 & 0.410 & -0.009 & 0.976 & 0.000 & 0.999 \\
\hline
\end{tabular}

Values are Pearson's correlation coefficients and associated $\mathrm{p}$ values. Values in bold indicate statistically significant correlation at a value of $p<0.05$.

${ }^{*} P<0.05,{ }^{* *} \mathrm{p}<0.005$.

ALT, alanine transaminase; AST, aspartate transaminase; BMI, body mass index; BP, blood pressure; CKD-EPI, CKD Epidemiology Collaboration; eGFR, estimated glomerular filtration rate; HbA1c, hemoglobin A1c; HDL, high-density lipoprotein; LDL, lowdensity lipoprotein; mtCOX-3, mitochondrial cytochrome-c oxidase 3; mtND-1, mitochondrial nicotinamide adenine dinucleotide dehydrogenase subunit-1; T2DM, type 2 diabetes mellitus.

surgery can improve metabolic parameters, and the mitochondrial dysfunction in patients with morbid obesity. Further studies are needed to investigate the long-term effect of bariatric surgery on metabolic dysfunction in patients with mild obesity, and patients without obesity with T2DM.

Contributors Each author was involved in, and contributed to, this manuscript. Study concept, study design and data collection: SoHK. Analysis of data and writing original draft: MS and SoHK. Study supervision: HK, HN, JSJ, DWB and SaHK. Protocol review and contribution to discussion: HJK., KS and HKP. All authors contributed to the writing of the manuscript. All authors reviewed the manuscript and approved the final version.

Funding This study was supported by the Bio \& Medical Technology Development Program of the National Research Foundation (NRF) funded by the Korean government (MSIT) (NRF-2019M3E5D3073102) and the Soonchunhyang University Research Fund

Competing interests None declared.

Patient consent for publication Not required.

Ethics approval The study protocol was approved by the Institutional Review Board (IRB Number 2015-11-020) of Soonchunhyang University Hospital. All participants provided written informed consent.

Provenance and peer review Not commissioned; externally peer reviewed.

Data availability statement Data are available on reasonable request. Deindentifed participants' data.

Open access This is an open access article distributed in accordance with the Creative Commons Attribution Non Commercial (CC BY-NC 4.0) license, which permits others to distribute, remix, adapt, build upon this work non-commercially, and license their derivative works on different terms, provided the original work is properly cited, appropriate credit is given, any changes made indicated, and the use is non-commercial. See: http://creativecommons.org/licenses/by-nc/4.0/.

ORCID iDs

Hyoungnae Kim http://orcid.org/0000-0002-5359-0214

Soon Hyo Kwon http://orcid.org/0000-0002-4114-4196

\section{REFERENCES}

1 Malik AN, Czajka A. Is mitochondrial DNA content a potential biomarker of mitochondrial dysfunction? Mitochondrion 2013;13:481-92.

2 Lee HK, Song JH, Shin CS, et al. Decreased mitochondrial DNA content in peripheral blood precedes the development of noninsulin-dependent diabetes mellitus. Diabetes Res Clin Pract 1998;42:161-7.

3 Gianotti TF, Sookoian S, Dieuzeide G, et al. A decreased mitochondrial DNA content is related to insulin resistance in adolescents. Obesity 2008;16:1591-5.

4 Hansen M, Lund MT, Gregers E, et al. Adipose tissue mitochondrial respiration and lipolysis before and after a weight loss by diet and RYGB. Obesity 2015;23:2022-9.

5 Heinonen S, Buzkova J, Muniandy M, et al. Impaired mitochondrial biogenesis in adipose tissue in acquired obesity. Diabetes 2015:64:3135-45.

6 Sacks J, Mulya A, Fealy CE, et al. Effect of Roux-en-Y gastric bypass on liver mitochondrial dynamics in a rat model of obesity. Physiol Rep 2018;6:e13600.

7 Nijhawan S, Richards W, O'Hea MF, et al. Bariatric surgery rapidly improves mitochondrial respiration in morbidly obese patients. Surg Endosc 2013;27:4569-73.

8 Kwon SH. Changes in kidney function markers after bariatric surgery in morbidly obese patients. Kidney Res Clin Pract 2020;39:115-7.

9 Bradley D, Conte C, Mittendorfer B, et al. Gastric bypass and banding equally improve insulin sensitivity and $\beta$ cell function. $J$ Clin Invest 2012;122:4667-74. 
10 Cummings DE, Rubino F. Metabolic surgery for the treatment of type 2 diabetes in obese individuals. Diabetologia 2018;61:257-64.

11 Madsen LR, Baggesen LM, Richelsen B, et al. Effect of Roux-en-Y gastric bypass surgery on diabetes remission and complications in individuals with type 2 diabetes: a Danish population-based matched cohort study. Diabetologia 2019;62:611-20.

12 Bankoglu EE. Impact of weight loss induced by gastric bypass or caloric restriction on. , 2016: 94, 208-17.

13 Whitaker RM, Stallons LJ, Kneff JE, et al. Urinary mitochondrial DNA is a biomarker of mitochondrial disruption and renal dysfunction in acute kidney injury. Kidney Int 2015;88:1336-44.

14 Eirin A, Saad A, Tang H, et al. Urinary mitochondrial DNA copy number identifies chronic renal injury in hypertensive patients. Hypertension 2016;68:401-10.

$15 \mathrm{Cao} \mathrm{H}, \mathrm{Wu} \mathrm{J}$, Luo J, et al. Urinary mitochondrial DNA: a potential early biomarker of diabetic nephropathy. Diabetes Metab Res Rev 2019;35:e3131.

$16 \mathrm{Bae} \mathrm{JH}$, Jo SI, Kim SJ, et al. Circulating cell-free mtDNA contributes to AIM2 inflammasome-mediated chronic inflammation in patients with type 2 diabetes. Cells 2019;8. doi:10.3390/cells8040328. [Epub ahead of print: 08 Apr 2019].

17 Lee H, Oh S, Yang W, et al. Bariatric surgery reduces elevated urinary mitochondrial DNA copy number in patients with obesity. $J$ Clin Endocrinol Metab 2019;104:2257-66.

18 Skuratovskaia D, Litvinova L, Vulf M, et al. From normal to obesity and back: the associations between mitochondrial DNA copy number, gender, and body mass index. Cells 2019;8. doi:10.3390/ cells8050430. [Epub ahead of print: 09 May 2019]

19 Levey AS, Stevens LA, Schmid CH, et al. A new equation to estimate glomerular filtration rate. Ann Intern Med 2009;150:604-12.

20 Rötig A, Munnich A. Genetic features of mitochondrial respiratory chain disorders. J Am Soc Nephrol 2003;14:2995-3007.

21 Eirin A, Saad A, Woollard JR, et al. Glomerular hyperfiltration in obese African American hypertensive patients is associated with elevated urinary Mitochondrial-DNA copy number. Am J Hypertens 2017;30:1112-9.

22 Verbeek J, Lannoo M, Pirinen E, et al. Roux-en-Y gastric bypass attenuates hepatic mitochondrial dysfunction in mice with nonalcoholic steatohepatitis. Gut 2015;64:673-83.

23 Chang AR, Chen Y, Still C, et al. Bariatric surgery is associated with improvement in kidney outcomes. Kidney Int 2016;90:164-71.

24 Lee S, Park S, Kwak MK, et al. Predictors of postoperative eGFR change and resolution of hyperfiltration in obese patients following bariatric surgery. Surg Obes Relat Dis 2017;13:1353-60.

25 Marseglia L, Manti S, D'Angelo G, et al. Oxidative stress in obesity: a critical component in human diseases. Int J Mol Sci 2014;16:378-400.

26 Bai J, Cervantes C, Liu J, et al. Dsba-L prevents obesity-induced inflammation and insulin resistance by suppressing the mtDNA release-activated cGAS-cGAMP-STING pathway. Proc Natl Acad Sci U S A 2017;114:12196-201. 